\title{
Assessment of the fear of COVID-19 and its impact on lung cancer screening participation among the Korean general population
}

\author{
Thuy Linh Duong ${ }^{1}$, Nayoung Lee ${ }^{2}$, Yonghyun Kim ${ }^{2}$, Yeol Kim ${ }^{1,2}$ \\ ${ }^{1}$ Department of Cancer Control and Population Health, Graduate School of Cancer Science and Policy, National Cancer Center, Goyang, Republic \\ of Korea; ${ }^{2}$ National Cancer Control Institute, National Cancer Center, Goyang, Republic of Korea \\ Contributions: (I) Conception and design: Y Kim; (II) Administrative support: Y Kim; (III) Provision of study materials or patients: Y Kim; (IV) \\ Collection and assembly of data: N Lee, Y Kim, Y Kim; (V) Data analysis and interpretation: TL Duong, Y Kim; (VI) Manuscript writing: All \\ authors; (VII) Final approval of manuscript: All authors. \\ Correspondence to: Yeol Kim, MD, PhD. National Cancer Control Institute, National Cancer Center, 809 Madu 1(il)-dong, Ilsandong-gu, Goyang-si, \\ Gyeonggi-do, Republic of Korea. Email: drheat@ncc.re.kr.
}

Background: We aimed to assess the fear of COVID-19 and how much it affected the behaviors of the general population towards cancer screening.

Methods: The Korean National Cancer Screening Survey is an annual population-based, cross-sectional survey to investigate cancer screening rates of five major malignancies. We extracted data on 3,557 cancerfree respondents aged $\geq 40$ years in 2020, including sociodemographic characteristics, comorbidities, family history of cancer, self-perceived general health status, attitudes towards screening, and fear of COVID-19 compared with lung cancer. We collected information on health check-ups participation including cancer screening with or without schedule during the pandemic and analyzed the participation rate according to the degree of fear of COVID-19.

Results: Among 3,557 respondents, 1,066 (29.97\%) people were more worried by COVID-19 than by lung cancer. 2,392 (67.25\%) did not participate in health check-ups, of which $573(24.0 \%)$ had a schedule for health check-ups but did not receive. We observed a significant increase $(\mathrm{P}<0.05)$ in the proportion of nonparticipation with schedule when the fear of COVID-19 exceeds lung cancer. In multivariate logistic analysis, the respondents with more fear of COVID-19 compared with lung cancer showed decreased likelihood of attendance in health check-ups (OR, 0.84; 95\% CI: 0.71-0.98).

Conclusions: A considerable proportion of the general population perceived more fear of COVID-19 than lung cancer, which significantly hampered their engagement in regular medical check-ups including cancer screening. This finding highlights the need for providing appropriate information to the target population of lung cancer screening to minimize disruption in cancer prevention activities.

Keywords: Cancer screening; coronavirus disease 2019 (COVID-19); lung cancer; fear

Submitted Sep 10, 2021. Accepted for publication Nov 11, 2021.

doi: $10.21037 /$ tlcr-21-746

View this article at: https://dx.doi.org/10.21037/tlcr-21-746

\section{Introduction}

In response to the Coronavirus Disease 2019 (COVID-19) crisis, prolonged lockdown, tight restrictions on gathering and travel have been imposed with numerous warning messages through broadcasts in effort to reduce disease transmission, which inevitably contributed to undesirable social and economic influences (1). During the year 2020, the pandemic has been found to be linked with a noteworthy rise of psychiatric and mental health issues, including anxiety, depression and post-traumatic stress symptoms in the general population $(2,3)$. Fear and worries about a new dangerous pathogen seem to hamper essential heath care seeking behaviors (4) as well as 
disease treatment compliance (5). Meanwhile, studies have revealed a sharp increase in not only COVID-19 related deaths but also deaths from other chronic and severe acute disorders $(6,7)$, suggesting the fear of coronavirus infection as additional barrier to health services should be adequately considered.

While the role of cancer screening has been thoroughly reported in tackling cancer mortality, there was already broad recognition of several challenges brought by the COVID-19 to cancer screening activities $(8,9)$. The decline in cancer screening has been documented since the beginning of the pandemic, resulting in delayed diagnosis $(10,11)$ and increased proportion of patients with advanced stages (12), which may eventually lead to dreadful effect on cancer mortality (13). According to recently updated GLOBOCAN estimates, lung cancer remains the leading cause of cancer death worldwide and the second most commonly diagnosed cancer, with approximately 1.8 million deaths and 2.2 million new diagnosed cases in 2020 (14). However, lung cancer screening participants often present with long-term smoking history and several underlying comorbidities (15), placing them at potentially higher risk of COVID-19 acquisition and poorer clinical outcomes (16), thereby possibly preventing them from routine screening activities during the pandemic.

The Korea National Cancer Screening Program (NCSP) (17), first launched in 1999, has targeted five major malignancies of the stomach, uterine cervix, breast, colorectum and liver. Annual lung cancer screening by low dose computed tomography (LDCT) has recently been added for high-risk groups (aged 55 to 74 years with smoking history of 30 pack-years or more) (18). Given current psychosocial threat in the midst of the pandemic, it is still unclear to what extent the fear of COVID-19 exists and how much it is responsible for the decline observed in cancer screening rates. An interesting question is whether people's fear of COVID-19 exceeds that of cancer and actually turns cancer screening into a risk-taking behavior. Utilizing a questionnaire survey in a representative sample of the Korean general public, we aim to assess the fear of COVID-19 in comparison with that of lung cancer and examine its association with the behaviors of respondents towards cancer screening, addressing how much COVID-19 affects population to participate in lung cancer screening.

We present the following article in accordance with the STROBE reporting checklist (available at https://dx.doi. org/10.21037/tlcr-21-746).

\section{Methods}

\section{Study population and data}

The Korean National Cancer Screening Survey (KNCSS) was a population-based, cross-sectional survey to investigate cancer screening rates for five major malignancies (gastric, liver, colorectal, breast, and cervix), conducted annually by the National Cancer Center in Korea since 2004. Survey subjects were recruited through a stratified, multistage random sampling procedure based on resident registration population according to geographical area, age, and sex. Details on sampling method was described in the previous studies $(19,20)$. Cancer-free men aged $\geq 40$ years and women aged $\geq 20$ years were eligible for participation according to the current protocols issued by the Korea National Cancer Screening Program (NCSP) (21). Informed consent to participate was obtained before the survey. This study was approved by the Institutional Review Board (IRB) of the National Cancer Center (IRB number: NCC20200041) and conducted in accordance with the Declaration of Helsinki (as revised in 2013). In 2020, data were collected from 05 August to 21 September through face-to-face interview with 43,241 people initially being contacted. After excluding 20,563 people who were absent at the time of household visit and 8,125 non-subjects, the response rate was $30.9 \%$ which is 4,500 out of 14,553 subjects. We asked them whether they participated in health check-ups (including cancer screening) as scheduled (Figure 1). Those aged under 40 years were all women targeted for cervical cancer screening (recommended for women from the age of 20 since 2016). As younger age people tend to have less concern and willingness to engage in health screening (22), we extracted data on only respondents aged 40 years and above for the final analysis to ensure comparable age strata between men and women.

\section{Measures}

Information was collected using a structured questionnaire on sociodemographic characteristics (including gender, age, education, household income); health-related status, including comorbidities (having any of the following conditions: hypertension, diabetes, tuberculosis, hepatitis B/ $\mathrm{C}$, liver cirrhosis, gastritis, ulcer, colon polyps, benign breast disease, uterine fibroids, hyperlipidemia), family history of cancer, self-perceived general health status (very good/ good/neutral/bad/very bad). Attitudes toward screening was assessed by the question "Do you think cancer screening 


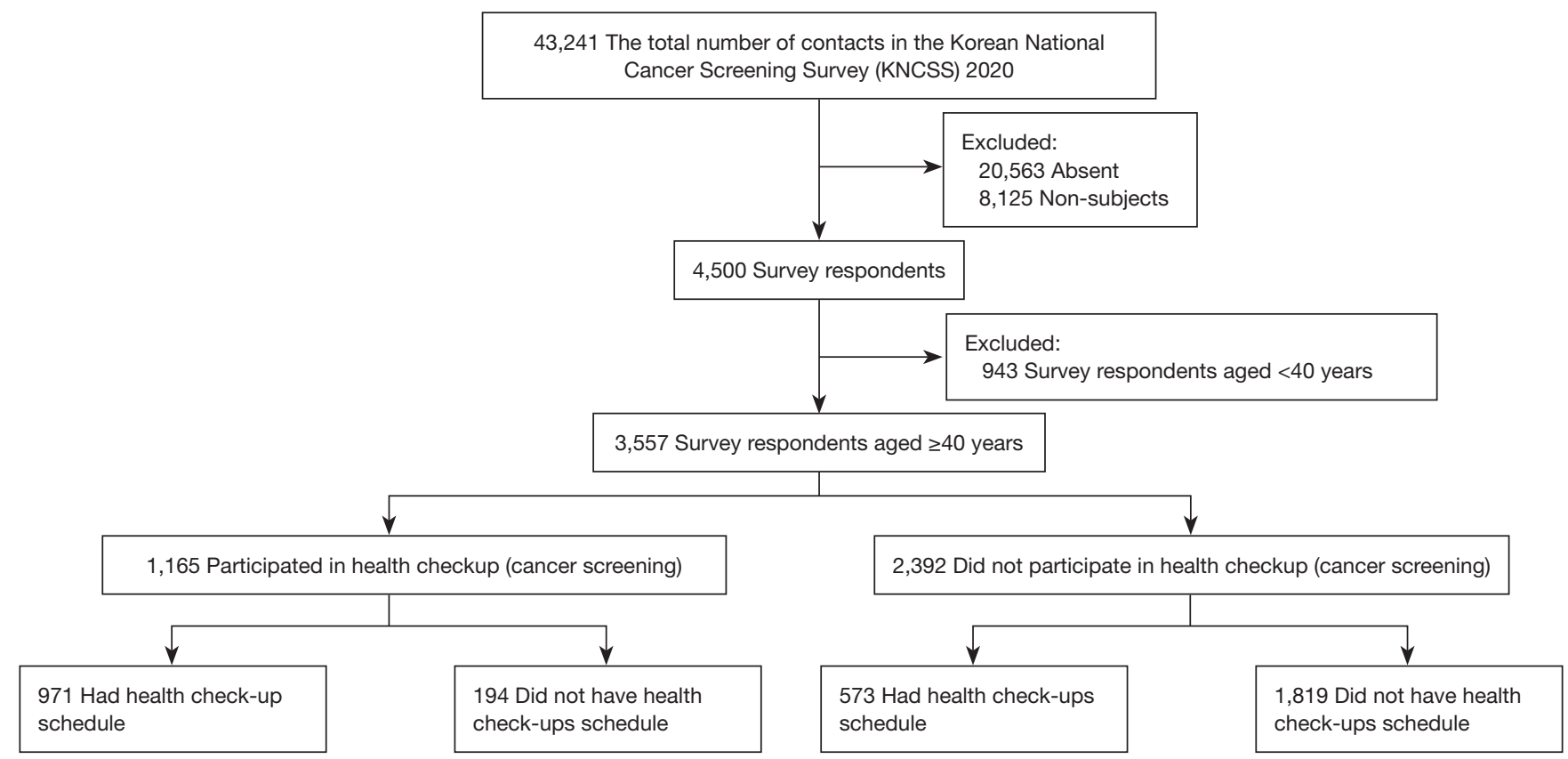

Figure 1 Flow of study participation and groups of participants by having health checkups with or without schedule. Absent: no one was available at the visiting household. Non-subjects: respondents were not subject to the target age group or were acknowledged that they were diagnosed with cancer before the survey interview. KNCSS, Korean National Cancer Screening Survey.

helps you find and cure cancer at early stage?" with four options for the answer: strongly agree, agree, disagree, and strongly disagree.

We added several specific questions to assess the impact of the COVID-19 pandemic on people's risk perception and health behavior changes, including "Which one is more fearful between coronavirus infection and lung cancer diagnosis in the current coronavirus spread?" where participants could rate their fear on a five-point scale towards coronavirus or lung cancer, then their answers were grouped into three main categories: more fear of coronavirus, neutral, or more fear of lung cancer. Outcome variable was defined by single question "Did you have any changes in health checkup (including cancer screening) due to COVID-19?"; the answer, which contained four categories: "participated as scheduled", "scheduled but did not participate", "did not schedule but participated", and "did not schedule and did not participate", was classified into those did and did not participate in cancer screening (with or without schedule) during the pandemic.

Korea lung cancer screening program was provided to high-risk groups composed of current and ex-smokers who have quit smoking within 15 years, aged 55 to 74 years with 30 pack-years or more of smoking-history. Data on smoking duration, intensity and past quit attempt were collected and analyzed for exploratory analysis on eligible lung cancer screening participants.

\section{Statistical analysis}

We performed descriptive analysis to explore study participants' characteristics, using Chi-square test to determine a significant difference between two categorical variables. Spearman rank test was used to examine the monotonic change in health check-ups non-participation across levels of Coronavirus fear in comparison with lung cancer. Univariate and multivariate logistic regression model were applied to identify factors associated with health check-ups participation. All statistical analysis were done by using STATA software version 15 (Stata Corp. L.P., College Station, TX). All $\mathrm{P}$ values were calculated from two-sided tests with significant level of $5 \%$.

\section{Results}

Among 3,557 respondents aged $\geq 40$ years in KNCSS 2020 (Table 1), 2,392 (67.25\%) did not participate in health checkups including cancer screening during the COVID-19 
Table 1 Characteristics of survey respondents $(\mathrm{N}=3,557)$

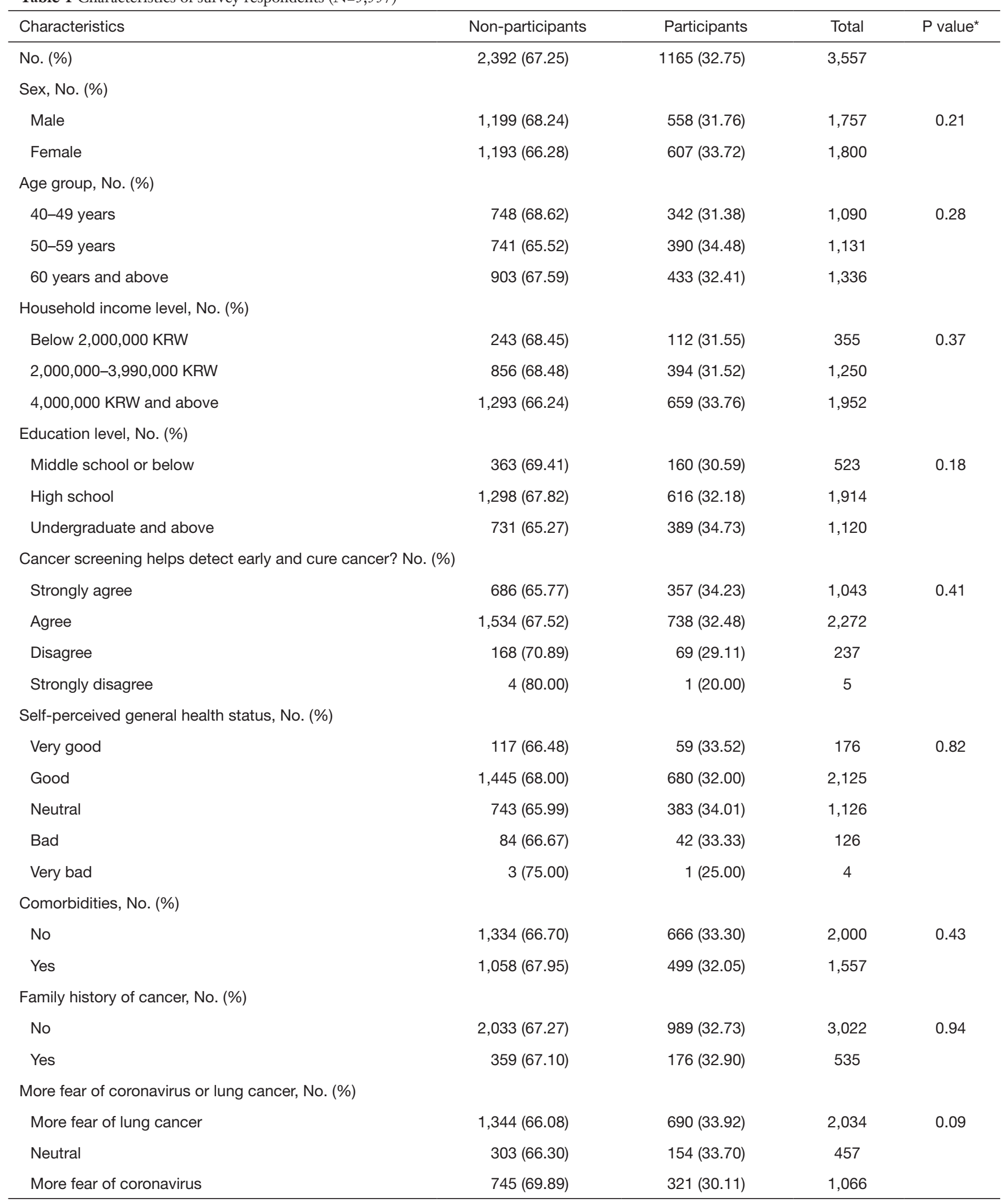

*, analyzed by Chi-square test. 

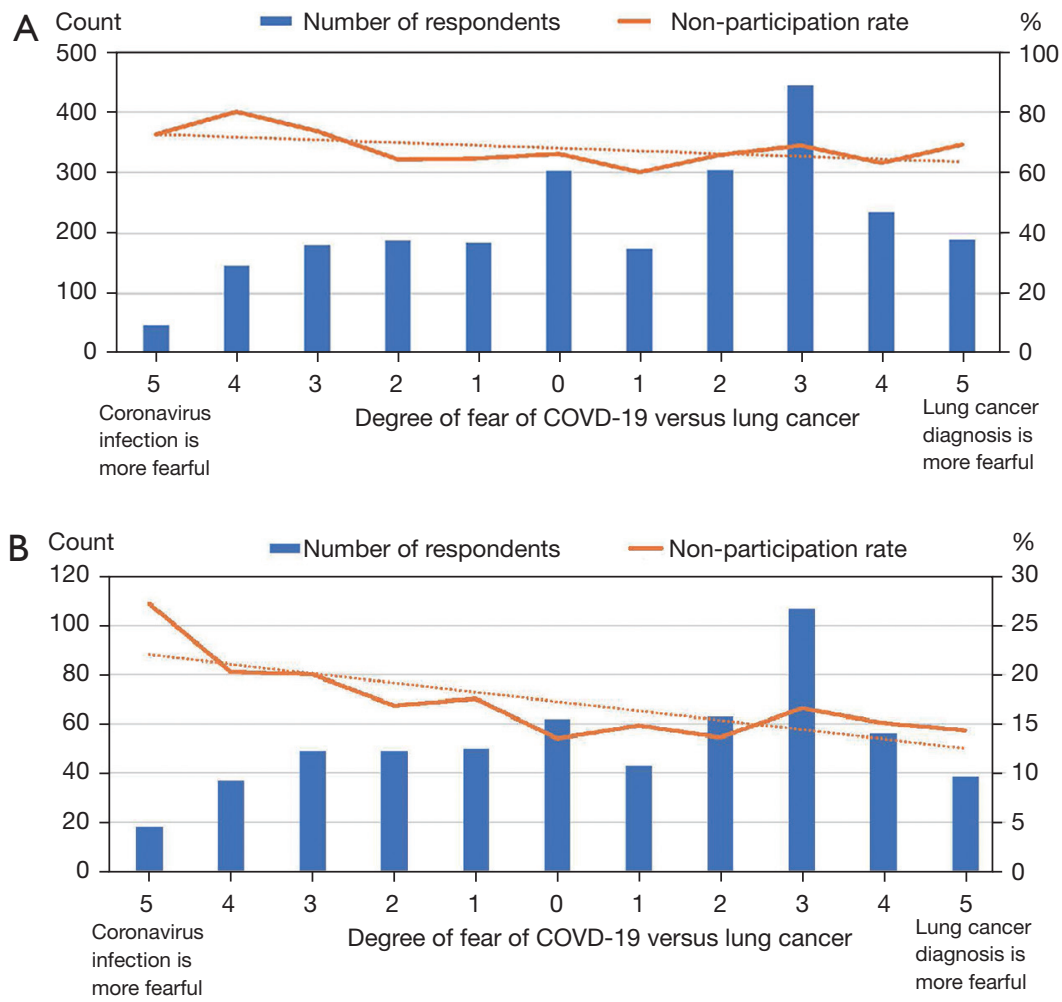

Figure 2 Trends in health check-ups non-participation by the fear of COVID-19 versus lung cancer. (A) Total non-participation in health check-ups. (B) Scheduled but did not participate.

pandemic, of which $573(24.0 \%)$ had a schedule for health check-ups but did not receive. The majority of respondents were high school graduates $(53.81 \%)$ and had household income level $\geq 4,000,000 \mathrm{KRW}$ (54.88\%). Notably, 1,066 (29.97\%) people were more worried by coronavirus than by lung cancer, while 457 (12.85\%) perceived equal fear of the two diseases and 2,034 (57.18\%) were more worried by lung cancer. $33.92 \%$ of those perceived more fear of lung cancer have participated in medical check-ups during the pandemic, whereas only $30.11 \%$ of those with more fear of COVID-19 have participated.

Figure 2 shows the trend in health check-ups nonparticipation across 11 levels of the fear of Coronavirus in comparison with lung cancer. The pattern in the rates of non-participation overall was not apparent, however we observed a statistically significant increase $(\mathrm{P}<0.05)$ in the percentage of those had check-ups schedule but did not participate when the fear of lung COVID-19 increases and exceeds that of lung cancer.

Variables associated with participation in health checkups are presented in Table 2. Older aged people were more likely to participate compared with those aged 40-49 years. Subjects with education level of undergraduate and above had higher odds of undergoing health check-ups than those with middle school level or below (OR, 1.38; 95\% CI: 1.02 1.87). Regarding our main interest, respondents with more fear of Coronavirus compared with lung cancer showed decreased likelihood of attendance in health check-ups (OR, 0.84; 95\% CI: 0.71-0.98) in multivariate model.

A total of 210 respondents were eligible for lung cancer screening program (Table 3). The excess fear of COVID-19 over lung cancer was present in 45 (21.43\%, versus $29.97 \%$ in the general public), of which only $15.56 \%$ (7 people) participated in health check-ups, whereas in the general public this figure was $30.11 \%$ (321 among 1,066 people with more fear of COVID-19).

\section{Discussion}

In this cross-sectional population-based study, we observed a significant upward trend in the rates of nonparticipation in pre-scheduled health check-ups when the 
Table 2 Factors associated with health check-ups participation in survey respondents by logistic regression analysis

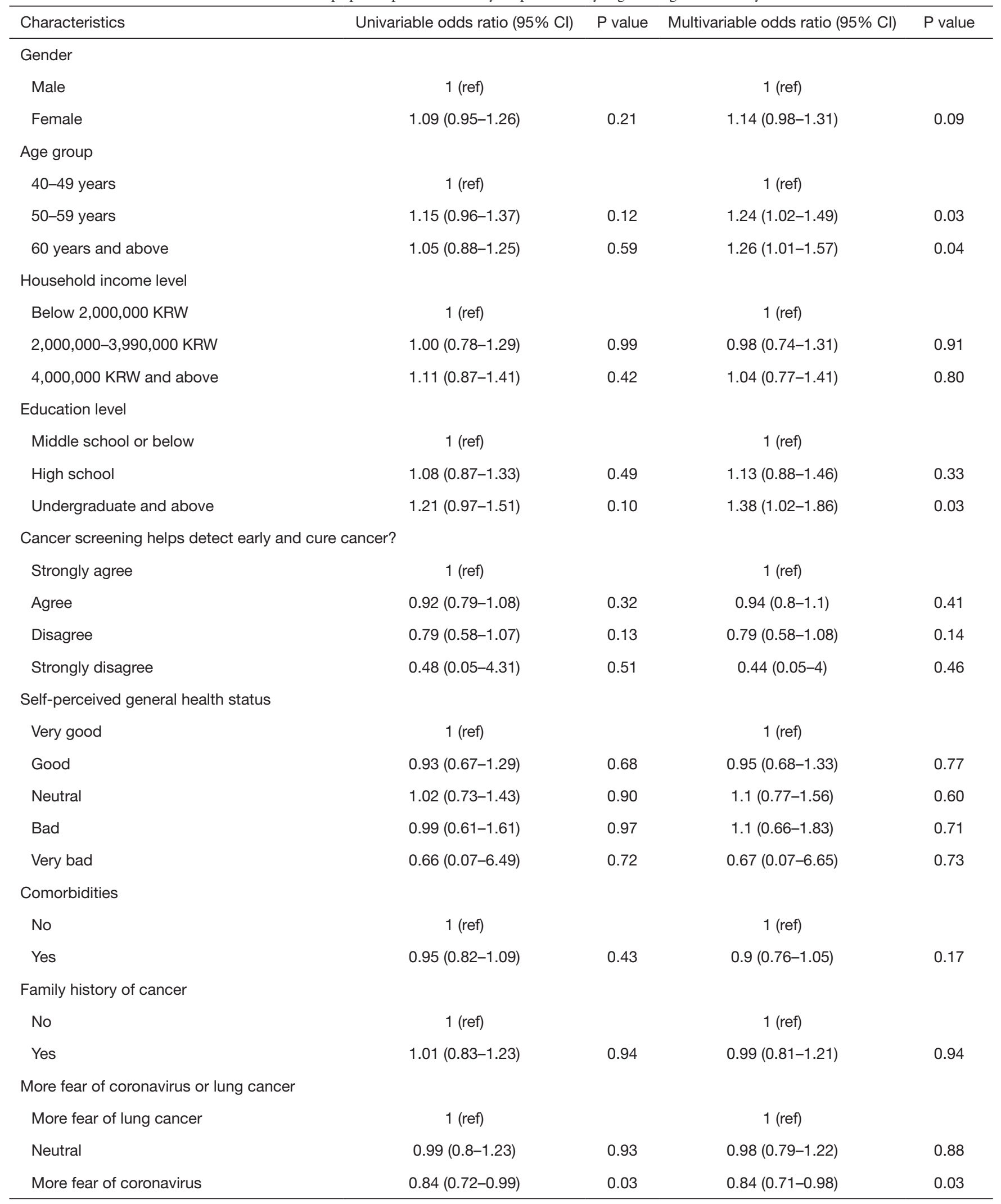


Table 3 Fear of COVID-19 versus lung cancer in lung cancer high-risk group

\begin{tabular}{lcccc}
\hline \multirow{2}{*}{ Fear of Coronavirus versus lung cancer } & \multicolumn{2}{c}{ Lung cancer high-risk population $(\mathrm{n}=210)$} & & \multicolumn{2}{c}{ Survey respondents $(\mathrm{n}=3,557)$} \\
\cline { 2 - 3 } Mealth check-ups participants, No. $(\%)$ & Total & Health check-ups participants, No. (\%) & Total \\
\hline Neutral & $7(15.56)$ & 45 & $321(30.11)$ & 1,066 \\
More fear of lung cancer & $6(28.57)$ & 21 & $154(33.7)$ & $690(33.92)$ \\
Total & $44(30.56)$ & 210 & $1,165(32.75)$ & 3,557 \\
$P$ value (Chi-square test) & $57(27.14)$ & 0.14 & 0.09 \\
\hline
\end{tabular}

fear of COVID-19 exceeds that of lung cancer, while the prevalence of this excess fear towards COVID-19 was approximately $30 \%$ in the general public. This excess fear of COVID-19 over lung cancer significantly hampered their engagement in screening as shown in multivariate logistic regression. These results raised great concerns about the direct impact on lung cancer screening when asymptomatic high-risk individuals tend to downplay their own risk of lung cancer development and attempt to avoid exposure to screening facilities.

Our finding confirmed hypothesis generated in previous investigations regarding the negative impact of COVID-19 fear on health seeking behaviors (23). While the spread of coronavirus has forced physicians to modify usual standards of care for non-covid patients (24), the fear of exposing themselves to coronavirus along with the thought "bothering physicians for non-COVID-19 related symptoms" have triggered people's reluctance to visit health facilities for routine or even emergent issues (25). Studies assessing the fear of COVID-19 and its impact have been done intensively in cancer patients because of their high vulnerability due to the immune deficiency induced by cancer and its treatment $(5,26)$, following by the development of specific communication strategies in this subgroup (27). However, studies investigating the fear in the general population and how it affected health screening are scarce. Utilizing a relatively representative sample of Korean general population, our survey question targeted a specific aspect of psychology under COVID-19 circumstance as people tended to weight their fear of a clinically relevant illness with the fear of coronavirus, affecting their decision to undergo or postpone medical procedures (28). A previous survey in Italy designed to assess this aspect in patients with lung cancer reported $21 \%$ of them were more worried about COVID-19 than their disease, mostly in long-term survivors (29). Meanwhile, nearly $30 \%$ of our survey respondents perceived a COVID-19 diagnosis was more fearful-a seemingly surprising proportion given the fact that lung cancer in Korea remains the most common and leading cause of death among cancer types, with approximately 18,000 deaths in 2017 (while the total deaths from COVID-19 in Korea stayed at around 900 on April 2020). Reasons for this perception could be diversified, involving one's knowledge of disease severity itself and possibly the fear related to risk of loved ones or socioeconomic consequences (30). However, it was not likely that people were not well-aware of lung cancer danger, but instead the accompanying COVID-19 infodemic, numerous warnings through broadcasts, unceasing text message alerts and prolonged social distancing stages might altogether aggravated psychological attacks in the public, causing such misperception and furthermore, the avoidance of disease screening. Though Korea has successfully flattened the curve in a timely manner with the aid of proactive and drastic strategies, fear and misperception of COVID-19 in the normalization state—as partly anticipated-persisted to a considerable extent in the public and significantly held back cancer prevention. From this perspective, we believe improvement of public's awareness on lung cancer prevention remains critical during the ongoing pandemic, and that public risk communication should be designed not to foster self-protective practices at the expense of exacerbating the fear of COVID-19, but instead to promote sustainable behavior change while reinforcing compliance with disease screening guidelines especially for lung cancer. In addition, psychological assessment and adequate consultation should be given special attention when operating cancer screening to promote accurate perception regarding disease risks and severity, particularly for lung cancer screening, and to prevent graver consequences as the 
pandemic is still far from over.

Lung cancer screening in high-risk population using LDCT has been endorsed to tackle lung cancer mortality in the two largest population-based clinical trials in the US and Europe $(31,32)$. Korea was the first country in the world to introduce nationwide lung cancer screening program, with $90 \%$ financial support from The National Health Insurance Service (NHIS). Although previously reported feasibility pilot study-The Korean Lung Cancer Screening Project (K-LUCAS)—showed promising results, several challenges remained affecting the effectiveness of the program including refusal of screening in highrisk group and poor compliance of follow-up diagnostic procedure among individuals with abnormal results (18). Furthermore, those with high socioeconomic status (SES) seemed to dominate among screening participants while most heavy smokers belong to low SES group, as does lung cancer risk (33). This raised concerns about access to lung cancer screening programs of low SES individuals and the need for strategies in effort to achieve high screening uptake especially in underserved groups as well as to improve follow-up compliance when implementing the screening program. This became even more challenging when the introduction of coronavirus has created more obstacles for lung cancer high-risk groups to receive and adhere to screening recommendations. Eligible screening subjects are of older age with long-term smoking history and usually more underlying comorbidities compared with the general population, thus might be placed at greater risk of infection and worse health complications in the pandemic. Even though only 45 among 210 eligible lung cancer screening participants in our study perceived more fear of COVID-19 ( $21.43 \%$, versus $29.97 \%$ in the general public), only $15.56 \%$ (7 people) of them participated in health checkups, whereas this figure for the total survey respondents was almost doubled at $30.11 \%$. This suggested that while the fear of contracting virus added another barrier to these vulnerable groups, underestimating lung cancer hazards compared with COVID-19 further discouraged them to seek help and ultimately lead to postponement of screening schedule. One study in the US reported that even after full reoperation of previous suspended screening program, the monthly number of participants remained low and the rate of those who did not show up or postpone screening schedule has significantly raised during COVID-19 period (34). A constant number of lung cancer diagnosis was observed before and after COVID-19 outbreak in a study conducted at three Korean hospitals ( $N=169$ in 2020, 166 in 2019, 139 in 2018 and 138 in 2017; $\mathrm{P}=0.605$ ), most likely due to the country's drastic screening of COVID-19 and proactive triaging of suspected patients, however the proportion of non-small cell lung cancer (NSCLC) patients with advanced stages significantly increased to $74.7 \%$ compared with the previous years $(57.9 \%$ in $2017,66.7 \%$ in 2018 , and $62.7 \%$ in 2019; $\mathrm{P}=0.011$ ) (12). Though several guidelines warranted a short-term delay (e.g., 6-12 months) in cancer screening because of the immediate risks related with the COVID-19 surge (35), as the pandemic timeline continues to extend following by huge backlog of patients with prolonged screening delay, we may end up inverting the progress achieved in recent years for lung cancer survival and mortality. A modelling study in UK estimated a 4.8-5.3\% increase in the number of lung cancer deaths up to 5 years after diagnosis, corresponding to $1,235(1,220-1,254)$ to 1,372 $(1,343-1,401)$ additional deaths as a result of a 12-month delay in diagnosis since the country's lockdown on March 2020 (13). Furthermore, impact of missing a cancer screening is not the same for every population, but could be amplified among minority subgroups (9). Therefore, under the stabilization state of COVID-19, lung cancer screening should still be prioritized and maintained to prevent downstream consequences of diagnosis delay on patients' health outcomes and mortality. Korea has also released its own guidelines for cancer care stating that cancer screening should not be delayed in healthy subjects who were not suspected of COVID-19 infection unless there is a shortage of medical resources (36). Appropriate health communication to the general public and high-risk groups has been left to play central role in preserving screening effectiveness.

Our study has some limitations. First, we could not establish causal relationships due to the cross-sectional nature. Second, all information were self-reported and retrospectively collected by trained interviewers, thus recall and interviewer biases might have occurred albeit fairly small as we collected information on recent health screening experience specified only during the pandemic. Lastly, we could not calculate exactly the proportion of the population who underwent specific cancer screening, particularly lung cancer screening, thus could not assess directly the impact of the fear and misperception on lung cancer screening participation. However, our findings provided important evidence for future strategies in efforts to normalize and improve screening uptake for 
lung cancer and possibly for other malignancies amid the pandemic. Future studies should be proceeded in depth to evaluate the performance of current lung cancer screening programs.

Research and countermeasures on COVID-19 has been conducted at an exceptional pace including the introduction of COVID-19 vaccines in addition to the emergence of novel coronavirus variants, thus information is still rapidly evolving, further challenging health communication messaging. Excess fear of COVID-19 over lung cancer discouraged at-risk individuals in attending cancer screening, which may eventually lead to delayed diagnosis, poorer health outcomes and elevated cancer mortality in the long run. Our findings emphasize the need to develop appropriate health communication approach amid the ongoing pandemic, particularly for high-risk individuals targeted in lung cancer screening, including distress and fear assessment as well as providing adequate information to promote accurate perceived susceptibility and reduce perceived barriers, thus could effectively normalize and enhance screening compliance among these vulnerable groups.

\section{Acknowledgments}

Funding: This study was supported by a grant from the National R\&D Program for Cancer Control and national health promotion fund, Ministry of Health and Welfare, Republic of Korea (2060820-1, 2060740-1), and a grant from National Cancer Center, Republic of Korea (1910200). The funding sources did not have any role in the study design, in the collection, analysis and interpretation of data, in the writing of the report, nor in the decision to submit the article for publication.

\section{Footnote}

Reporting Checklist: The authors have completed the STROBE reporting checklist. Available at https://dx.doi. org/10.21037/tlcr-21-746

Data Sharing Statement: Available at https://dx.doi. org/10.21037/tlcr-21-746

Conflicts of Interest: All authors have completed the ICMJE uniform disclosure form (available at https://dx.doi. org/10.21037/tlcr-21-746). The authors have no conflicts of interest to declare.
Ethical Statement: The authors are accountable for all aspects of the work in ensuring that questions related to the accuracy or integrity of any part of the work are appropriately investigated and resolved. Informed consent to participate was obtained before the survey. This study was approved by the Institutional Review Board (IRB) of the National Cancer Center (IRB number: NCC20200041) and conducted in accordance with the Declaration of Helsinki (as revised in 2013).

Open Access Statement: This is an Open Access article distributed in accordance with the Creative Commons Attribution-NonCommercial-NoDerivs 4.0 International License (CC BY-NC-ND 4.0), which permits the noncommercial replication and distribution of the article with the strict proviso that no changes or edits are made and the original work is properly cited (including links to both the formal publication through the relevant DOI and the license). See: https://creativecommons.org/licenses/by-nc-nd/4.0/.

\section{References}

1. Nicola M, Alsafi Z, Sohrabi C, et al. The socio-economic implications of the coronavirus pandemic (COVID-19): A review. Int J Surg 2020;78:185-93.

2. Bao Y, Sun Y, Meng S, et al. 2019-nCoV epidemic: address mental health care to empower society. Lancet 2020;395:e37-8.

3. Vindegaard N, Benros ME. COVID-19 pandemic and mental health consequences: Systematic review of the current evidence. Brain Behav Immun 2020;89:531-42.

4. Lazzerini M, Barbi E, Apicella A, et al. Delayed access or provision of care in Italy resulting from fear of COVID-19. Lancet Child Adolesc Health 2020;4:e10-1.

5. Karacin C, Bilgetekin I, B Basal F, et al. How does COVID-19 fear and anxiety affect chemotherapy adherence in patients with cancer. Future Oncol 2020;16:2283-93.

6. Woolf SH, Chapman DA, Sabo RT, et al. Excess Deaths From COVID-19 and Other Causes, March-July 2020. JAMA 2020;324:1562-4.

7. Raleigh VS. Tackling UK's mortality problem: covid-19 and other causes. BMJ 2020;369:m2295.

8. Lang M, Yeung T, Shepard JO, et al. Operational Challenges of a Low-Dose CT Lung Cancer Screening Program During the Coronavirus Disease 2019 Pandemic. Chest 2021;159:1288-91.

9. Cancino RS, Su Z, Mesa R, et al. The Impact of 
COVID-19 on Cancer Screening: Challenges and Opportunities. JMIR Cancer 2020;6:e21697.

10. Kaufman HW, Chen Z, Niles J, et al. Changes in the Number of US Patients With Newly Identified Cancer Before and During the Coronavirus Disease 2019 (COVID-19) Pandemic. JAMA Netw Open 2020;3:e2017267.

11. Patt D, Gordan L, Diaz M, et al. Impact of COVID-19 on Cancer Care: How the Pandemic Is Delaying Cancer Diagnosis and Treatment for American Seniors. JCO Clin Cancer Inform 2020;4:1059-71.

12. Park JY, Lee YJ, Kim T, et al. Collateral effects of the coronavirus disease 2019 pandemic on lung cancer diagnosis in Korea. BMC Cancer 2020;20:1040.

13. Maringe C, Spicer J, Morris M, et al. The impact of the COVID-19 pandemic on cancer deaths due to delays in diagnosis in England, UK: a national, population-based, modelling study. Lancet Oncol 2020;21:1023-34.

14. Sung H, Ferlay J, Siegel RL, et al. Global Cancer Statistics 2020: GLOBOCAN Estimates of Incidence and Mortality Worldwide for 36 Cancers in 185 Countries. CA Cancer J Clin 2021;71:209-49.

15. Howard DH, Richards TB, Bach PB, et al. Comorbidities, smoking status, and life expectancy among individuals eligible for lung cancer screening. Cancer 2015;121:4341-7.

16. Oh TK, Song IA. Impact of coronavirus disease-2019 on chronic respiratory disease in South Korea: an NHIS COVID-19 database cohort study. BMC Pulm Med 2021;21:12.

17. Kim Y, Jun JK, Choi KS, et al. Overview of the National Cancer screening programme and the cancer screening status in Korea. Asian Pac J Cancer Prev 2011;12:725-30.

18. Lee J, Kim Y, Kim HY, et al. Feasibility of implementing a national lung cancer screening program: Interim results from the Korean Lung Cancer Screening Project (K-LUCAS). Transl Lung Cancer Res 2021;10:723-36.

19. Lee HY, Park EC, Jun JK, et al. Trends in socioeconomic disparities in organized and opportunistic gastric cancer screening in Korea (2005-2009). Cancer Epidemiol Biomarkers Prev 2010;19:1919-26.

20. Suh M, Choi KS, Park B, et al. Trends in Cancer Screening Rates among Korean Men and Women: Results of the Korean National Cancer Screening Survey, 20042013. Cancer Res Treat 2016;48:1-10.

21. Shim BY. Cancer Screening Guidelines in Korea. Korean J Med 2016;90:224-30.

22. Deeks A, Lombard C, Michelmore J, et al. The effects of gender and age on health related behaviors. BMC Public Health 2009;9:213.

23. Stolow JA, Moses LM, Lederer AM, et al. How Fear Appeal Approaches in COVID-19 Health Communication May Be Harming the Global Community. Health Educ Behav 2020;47:531-5.

24. Rosenbaum L. The Untold Toll - The Pandemic's Effects on Patients without Covid-19. N Engl J Med 2020;382:2368-71.

25. Rubin R. COVID-19's Crushing Effects on Medical Practices, Some of Which Might Not Survive. JAMA 2020;324:321-3.

26. Vanni G, Materazzo M, Pellicciaro M, et al. Breast Cancer and COVID-19: The Effect of Fear on Patients' Decisionmaking Process. In Vivo 2020;34:1651-9.

27. Gharzai LA, Resnicow K, An LC, et al. Perspectives on Oncology-Specific Language During the Coronavirus Disease 2019 Pandemic: A Qualitative Study. JAMA Oncol 2020;6:1424-8.

28. Armellini E, Repici A, Alvisi C, et al. Analysis of patients attitude to undergo urgent endoscopic procedures during COVID-19 outbreak in Italy. Dig Liver Dis 2020;52:695-9.

29. Catania C, Spitaleri G, Del Signore E, et al. Fears and Perception of the Impact of COVID-19 on Patients With Lung Cancer: A Mono-Institutional Survey. Front Oncol 2020;10:584612.

30. Mertens G, Gerritsen L, Duijndam S, et al. Fear of the coronavirus (COVID-19): Predictors in an online study conducted in March 2020. J Anxiety Disord 2020;74:102258.

31. de Koning HJ, van der Aalst CM, de Jong PA, et al. Reduced Lung-Cancer Mortality with Volume CT Screening in a Randomized Trial. N Engl J Med 2020;382:503-13.

32. National Lung Screening Trial Research Team; Aberle DR, Adams AM, et al. Reduced lung-cancer mortality with low-dose computed tomographic screening. N Engl J Med 2011;365:395-409.

33. Schütte S, Dietrich D, Montet X, et al. Participation in lung cancer screening programs: are there gender and social differences? A systematic review. Public Health Rev 2018;39:23.

34. Van Haren RM, Delman AM, Turner KM, et al. Impact of the COVID-19 Pandemic on Lung Cancer Screening Program and Subsequent Lung Cancer. J Am Coll Surg 2021;232:600-5.

35. Mazzone PJ, Gould MK, Arenberg DA, et al. Management 
of Lung Nodules and Lung Cancer Screening During the COVID-19 Pandemic: CHEST Expert Panel Report.

Chest 2020;158:406-15.

Cite this article as: Duong TL, Lee N, Kim Y, Kim Y. Assessment of the fear of COVID-19 and its impact on lung cancer screening participation among the Korean general population. Transl Lung Cancer Res 2021;10(12):4403-4413. doi: $10.21037 /$ tlcr-21-746
36. Lee JB, Jung M, Kim JH, et al. Guidelines for Cancer Care during the COVID-19 Pandemic in South Korea. Cancer Res Treat 2021;53:323-9. 